\title{
SÍTIOSASSISTENCIAIS EM UNIDADE DE TERAPIA INTENSIVA E RELAÇÃO DO NURSING ACTIVITIES SCORE COM A INFECÇÃO HOSPITALAR
}

Assistance sites in the intensive care unit and the relation from nursing activities score with the hospital infection.

Sitios asistenciales en la unidad de terapia intensiva y la relación de nursing activities score con la infección hospitalaria

\section{RESUMO}

O Sistema de Classificação de Pacientes tornou-se essencial na prática gerencial e assistencial do enfermeiro. Este estudo analisou a implantação de Sítios Assistenciais na Unidade de Terapia Intensiva Adulto como forma de organização e classificação de pacientes, bem como suas implicações na qualidade do cuidado de acordo com o Nursing Activities Score e a relação com a Infecção Hospitalar. Trata-se de um estudo quantitativo, prospectivo, descritivo e transversal. A coleta foi realizada de julho a outubro de 2010. A amostra foi constituída por 214 pacientes, a maioria do sexo masculino, de neurocirurgia e com idade média de 57 anos. 0 Nursing Activities Score médio foi de 71,72\%. Com relação à Infecção Hospitalar antes e após a implantação, houve redução nas taxas de pneumonia, porém, a carga de trabalho de enfermagem permaneceu constante. Ficou evidenciada a importância da utilização do Nursing Activities Score como indicador e da implantação de novas formas de classificação de pacientes para melhorar a organização da assistência.

Palavras-chave: Carga de trabalho. Enfermagem. Organização e administração. Unidades de Terapia Intensiva.

\begin{abstract}
The Patient Classification System has become essential concerning to the practice in management and care from a nurse. This study analyzed the implementation of Assistance Sites in an intensive care unit for adults as a way of organization and classification of patients, as well as the impact of this process on the quality of care according to the Nursing Activities Score and the relation with the Hospital Infection. This is a quantitative, prospective, descriptive and transversal study. The data collection was realized from July until October 2010. The sample was consisted of 214 patients, mostly male, neurosurgical and with a mid age of 57 years. The NAS was on the average of $71.72 \%$. Regarding the Hospital Infection before and after implantation, there was a reduction in the rates of pneumonia. However, the nursing workload remained the same. Moreover, It was evident the importance of using the Nursing Activities Score and the implementation of new ways for classification of patients to improve the organization of the care.
\end{abstract}

Keywords: Workload. Nursing. Organization and Administration. Intensive Care Units

\section{Resumen}

El Sistema de Clasificación de Pacientes se tornó esencial para la práctica gerencial y asistencial del enfermero. Este estudio analizó la implantación de Sitios Asistenciales en la Unidad de Terapia Intensiva Adulta como forma de organización y clasificación de pacientes, bien como sus implicaciones en la calidad del cuidado de acuerdo con el Nursing Activities Score y la relación con Infección Hospitalaria. Se trata del estudio cuantitativo, prospectivo, descriptivo y transversal. La colección de datos fue realizada de julio a octubre de 2010. La muestra fue constituida por 214 pacientes, la mayoría hombres, neurocirúrgicos y con edad media de 57 años. El Nursing Activities Score medio fue 71,72\%. Con relación a la Infección Hospitalaria hubo reducción en los niveles de Neumonía, sin embargo, la carga de trabajo de enfermería permaneció constante. Quedó evidente la importancia del uso del NAS como indicador y la implantación de nuevas formas de clasificación de pacientes para mejorar la organización de la asistencia.

Palabras clave: Carga de trabajo. Enfermería. Organización y administración. Unidades de Cuidados Intensivos

\footnotetext{
${ }^{1}$ Enfermeira Mestre da Unidade de Terapia Intensiva Adulto do HC- Botucatu - SP. Brasil. E-mail: claudia.cyrino@yahoo.com.br; ${ }^{2}$ Professora Doutora do Departamento de Enfermagem da Faculdade de Medicina de Botucatu - SP. Brasil. E-mail: mqueiroz@fmb.unesp.br.
} 


\section{INTRODUÇÃO}

As Unidades de Terapia Intensivas (UTI) destinam-se ao atendimento de pacientes em estado agudo ou crítico, mas passíveis de recuperação, que requerem assistência médica e de enfermagem especializadas e ininterruptas. Nesse contexto, um dos desafios dos gestores dessas unidades é adequar a equipe de enfermagem às novas tecnologias e às demandas de cuidados exigidas pelos pacientes ${ }^{1}$.

Porém, a inadequação numérica e qualitativa dos recursos de enfermagem, principalmente em uma UTI, lesa os usuários no seu direito de assistência à saúde livre de riscos e pode comprometer a instituição pelas falhas ocorridas devido à sobrecarga de trabalho e à deficiência da qualidade da assistência prestada'.

Os modelos adotados para quantificação de pessoal de enfermagem têm passado, desde a década de 30, por um processo evolutivo. A mais recente evolução ocorreu com a introdução do Sistema de Classificação de Pacientes (SCP) como norteador do dimensionamento de pessoal, em 1961².

O SCP pode ser conceituado como a identificação e classificação de pacientes em grupos de cuidados ou categorias, e a quantificação dessas categorias como uma medida dos esforços de enfermagem requeridos. Dessa forma, a utilização de sistema de classificação possibilita agrupar os pacientes por complexidade assistencial, observar o perfil de cada grupo ou categoria previamente estabelecida; distribuir os leitos para atendimento da demanda por grupo de pacientes; realocar recursos materiais e humanos; detalhar a dinâmica operacional do sistema e reorientar a equipe envolvida no processo assistencial ${ }^{2,3}$.

Ele foi adaptado em 1994 à realidade brasileira e vem tornando-se essencial na prática gerencial de enfermagem, já que proporciona informações para o processo de tomada de decisões, para alocação de recursos humanos, monitorização da produtividade, verificação dos custos da assistência de enfermagem, enfim, para a organização e planejamento da assistência ${ }^{3}$.

Hoje existem várias propostas de SCP influenciadas por aspectos operacionais, práticas médicas e padrões assistenciais próprios de cada instituição. Portanto, sob o ponto de vista da enfermagem, o SCP ideal é aquele que utiliza instrumentos que permitam resultado seguro para a avaliação dos pacientes e da unidade Assim, observa-se, na prática, que não há total aplicabilidade dos sistemas de classificação já existentes, mas a preocupação da elaboração de novos instrumentos que melhor enquadram-se nos padrões específicos de cada instituição $0^{4}$.

Um recente instrumento utilizado nas UTIs como possibilidade de sistematizar e gerenciar o cuidado com qualidade é o Nursing Activities Score (NAS). Este aplicativo, desde 2002, quando foi traduzido e validado no Brasil, proporciona condições para justificar e dimensionar a equipe de enfermagem de acordo com a carga de trabalho, garantindo a melhora da assistência já que uma equipe superdimensionada torna-se mais cara e uma equipe reduzida pode implicar a diminuição da eficácia de cuidados, o que prolonga a hospitalização e aumenta o custo do tratamento do paciente ${ }^{5,6}$.

0 NAS é um instrumento sistemático, na forma de score, e consta de sete grandes categorias e 23 itens. Cada item possui uma pontuação; portanto, o escore atribuído a um paciente resulta da soma das pontuações dos itens que correspondem às necessidades de assistência direta e indireta dos pacientes. Portanto, quando os pontos se totalizam 100, interpreta-se que o paciente requereu $100 \%$ do tempo de um profissional de enfermagem no seu cuidado nas últimas 24 horas, podendo atingir no máximo 176,8\%,

$\mathrm{Na}$ UTI onde foi realizado esse estudo existe um aplicativo informatizado com o NAS, construído no ano de 2008; desde então o score passou a ser utilizado diariamente, agora eletronicamente compilado, pelas enfermeiras assistenciais da unidade para todos os pacientes admitidos. Este instrumento faz parte do processo de trabalho da UTI, colaborando para o dimensionamento e gerenciamento da assistência nesta unidade ${ }^{7}$.

Juntamente com o NAS, também fazem parte do processo de trabalho dessa unidade os Sítios Assistenciais, como um novo sistema de organização e classificação de pacientes. Assim, no cenário da pesquisa, foi reorganizada a alocação de pacientes conforme suas patologias, doenças crônicas preexistentes, pacientes em pré ou em pósoperatórios, pacientes crônicos, dias de internação na UTI e dias provenientes de outros locais do mesmo hospital.

Após essa proposta, outro aspecto a ser estudado foi a redução ou não dos índices de Infecção Hospitalar ( $(\mathrm{H})$. Avaliouse a separação dos pacientes recém-admitidos na UTI, em pósoperatório ou não, dos pacientes com mais dias de internação, o que poderia contribuir para o aumento dos procedimentos, uso de dispositivos cada vez mais invasivos conforme 0 prognóstico de cada paciente, os antimicrobianos e 0 aparecimento de micro-organismos multirresistentes.

Diante do interesse em avaliar esse novo processo de trabalho da equipe de enfermagem foi que se propôs o estudo que tem o seguinte objetivo: analisar a implantação de Sítios Assistenciais na UTI como forma de organização da assistência, a carga de trabalho de enfermagem por meio do NAS e a relação das taxas de Infecção Hospitalar antes e após essa nova implantação.

\section{MÉTODO}

Trata-se de um estudo quantitativo, prospectivo, descritivo e transversal sobre a implantação de Sítios Assistenciais na UTI Adulto de um Hospital Escola como um modelo de organização e de classificação de pacientes.

Os 15 leitos dessa UTI Adulto foram divididos em Sítios Assistenciais chamados de Células. A primeira seria a Célula 
de Isolamento, com quatro leitos, utilizada para pacientes com patologias que necessitam de isolamento por gotículas, aerossóis ou aqueles que necessitam de hemodiálise, já que nesses quartos encontram-se canalização com água de diálise; a segunda seria a Célula de Pós-Operatório, com cinco leitos, onde são internados principalmente pacientes para o pré ou pós-operatório de cirurgias eletivas ou de emergências, ou aqueles pacientes com entrada pelo Pronto-Socorro e que necessitam de cuidados intensivos; e na terceira Célula, com seis leitos divididos conforme a demanda da unidade, denominada Longa Permanência, são internados os pacientes crônicos, isto é, aqueles que permanecem na UTI por mais de seis dias e que, tendo condição, para o transporte são transferidos para tal Célula, assim como aqueles pacientes que estavam internados em outra enfermaria, que necessitam de cuidados intensivos e que supostamente estão potencialmente contaminados.

Cada Célula é composta por uma enfermeira assistencial e dois técnicos de enfermagem que permanecem fixos nesses locais por dez dias para depois passarem para outra Célula por mais dez dias, e assim por diante. Esta Célula somente é trocada de componente (profissionais) após um ou dois meses, com o objetivo de estreitar o contato pessoal entre os membros da equipe e os pacientes. Há também, em cada plantão, mais um técnico de enfermagem, chamado de volante, que assume os cuidados na Célula que apresenta a maior carga de trabalho, evidenciado pelo NAS.

Foram utilizados dois indicadores: a Infecção Hospitalar e a carga de trabalho de enfermagem. Os índices de Infecção Hospitalar foram descritos em dois momentos. 0 primeiro refere-se aos índices pregressos, em um período de seis meses antes da implantação dos Sítios Assistenciais de novembro de 2008 a abril de 2009; o segundo momento apresenta os índices de infecção seis meses após a nova implantação, de junho a novembro de 2009.
A carga de trabalho de enfermagem foi coletada diariamente por meio de um aplicativo (software), patente $\mathrm{n}^{\circ}$ RS11724-4, com o conteúdo do NAS. Ela foi avaliada de forma retrospectiva em cada Sítio Assistencial ${ }^{7,8}$.

0 projeto foi encaminhado para o Comitê de Ética e Pesquisa da Faculdade de Medicina de Botucatu-UNESP tendo como parecer o $n^{\circ}$ OF. 257/2010 - CEP.

A coleta de dados foi realizada pela pesquisadora no período de 1 de julho a 21 de outubro de 2010. A amostra constituiu-se por 214 pacientes, e as variáveis avaliadas foram: idade, sexo, especialidade, tempo de internação, desfecho, NAS e Sítio Assistencial obtidas diretamente da ficha de controle e prontuário dos pacientes. Estes dados foram eletronicamente compilados em uma planilha no sistema Statistical Analysis System (SAS) for windows, versão 9.2. Inicialmente foi realizada uma estatística descritiva dos dados com frequências e porcentagens, média, desvio-padrão, mediana, mínimo e máximo.

Para verificar a relação entre o NAS, a idade, o tempo de internação e verificar a diferença entre o desfecho dos pacientes, foi realizada uma correlação de Person e análise de variância (ANOVA).

Para a comparação da variável NAS no tempo e nos Sítios Assistenciais, foi utilizado um Modelo Misto com Medidas Repetidas no tempo, com interação tempo versus Sítios Assistenciais, ajustada por Tukey, por meio do PRO MIXED do programa SAS. Foi considerado $p<0,05$ como nível de significância para todos os testes estatísticos.

\section{RESULTADOS}

Dos 214 pacientes internados nesse período, $29 \%$ eram de especialidades clínicas e $71 \%$ de especialidades cirúrgicas, a maioria neurocirúrgicas e gastrocirúrgicas, conforme observase na Tabela 1.0 sexo masculino foi o de maior percentual, com $63 \%$ dos pacientes.

Tabela 1. Perfil dos pacientes segundo as variáveis categorizadas como sexo, especialidade, desfecho. Botucatu, 2010

\begin{tabular}{lll}
\hline Variáveis categorizadas & Frequência & \% \\
\hline Sexo & 135 & 63,08 \\
M & 79 & 36,91 \\
F & & \\
Especialidade $(n=20)$ & 59 & 27,57 \\
& & 18,69 \\
NCR & 40 & 11,21 \\
GCR & 24 & \\
CMG & & \\
Desfecho & 144 & 67,29 \\
& 58 & 27,1 \\
Alta & 12 & 5,6 \\
Óbito & & \\
AD & & \\
\hline Legenda: $M$ = masculino, $F=$ feminino; $N C R=$ neurocirurgia, GCR = gastrocirurgia, $C M G=$ clínica médica \\
geral; AD = data a definir.
\end{tabular}


Também na Tabela 1 observa-se que tiveram alta $67 \%$ dos pacientes; $27 \%$ foram a óbito e $6 \%$ dos pacientes estavam internados na UTI no final da coleta dos dados, não sendo verificado seu desfecho.

$\mathrm{Na}$ Tabela 2 conclui-se que a média da idade dos pacientes internados na UTI nesse período foi de
57 anos, sendo a idade mínima de 16 anos no mês de outubro e a máxima de 92 anos nos meses de julho e agosto. Quanto ao tempo de internação médio no período de coleta dos dados, foi de 9 dias, sendo o mínimo de um dia nos quatro meses analisados e máximo de 93 dias.

Tabela 2. Estatística descritiva dos pacientes quanto a idade, tempo de internação e o NAS. Botucatu, 2010

\begin{tabular}{llllll}
\hline Variáveis quantitativas & Média & Desvio-padrão & Mediana & Mínimo & Máximo \\
\hline Idade & 56,84 & 18,56 & 60 & 16 & 92 \\
TI & 9,29 & 13,3 & 4 & 1 & 93 \\
NAS & 71,72 & 10,39 & 69,79 & 48,2 & 109,1 \\
\hline
\end{tabular}

Legenda: $\mathrm{TI}=$ tempo de internação.

O NAS médio encontrado nesse estudo foi de $72 \%$ com o mínimo de $48 \%$ e o máximo de 109\%. Ele apresentou uma correlação negativa com o tempo de internação $(-0,23)$; portanto, os pacientes que apresentaram menor tempo de internação tinham maior carga de trabalho de enfermagem. Quanto à idade dos pacientes, não houve essa diferença estatisticamente significativa para afirmar que esta variável teve influência na carga de trabalho.

Os pacientes com o desfecho óbito foram os que apresentaram um maior NAS, $80 \%$, em relação aos que receberam alta, $68 \%$, representado por $p<0,05$.
Conforme se verifica na Tabela 3, entre os Sítios Assistenciais não houve diferença estatisticamente significativa entre os meses analisados. Já em relação aos meses, no mês de julho e agosto, houve diferença estatisticamente significativa entre a Célula do Isolamento (I) e de Longa Permanência (LP). No mês de setembro essa diferença foi encontrada entre as Células de Longa Permanência e do Pós-Operatório (PO), e no mês de outubro não houve diferença estatisticamente significativa entre os Sítios Assistenciais.

Tabela 3. Comparações do NAS no tempo e nos Sítios Assistenciais. Botucatu, 2010

\begin{tabular}{llllll}
\hline Sítios Assistenciais & Julho & Agosto & Setembro & Outubro & $\mathbf{P}(\mathbf{p}<0,05)$ \\
\hline I & $71,58 \mathrm{Aa}$ & $72,43 \mathrm{Aa}$ & $70,82 \mathrm{Aab}$ & $68,49 \mathrm{Aa}$ & \\
LP & $64,45 \mathrm{Bb}$ & $66,71 \mathrm{Bb}$ & $66,36 \mathrm{Ba}$ & $66,22 \mathrm{Ba}$ & \\
P0 & $68,01 \mathrm{Cab}$ & $68,29 \mathrm{Cab}$ & $72,51 \mathrm{Cb}$ & $70,7 \mathrm{Ca}$ & \\
\hline
\end{tabular}

$\mathrm{P}<0,05$ Leqenda: letra maiúscula $(A, B, C)=$ horizontal; letra minúscula $(a, b)=$ vertical

Na Tabela 4 observa-se uma redução nos índices de pneumonia associada à ventilação mecânica e um aumento nas taxas de infecção do trato urinário relacionado à sonda vesical de demora e de infecção de corrente sanguínea relacionada ao uso de cateter venoso central no período pós-implantação dos Sítios Assistenciais, de junho a novembro de 2009.

Tabela 4. Incidências de IRAS na UTI Central Adulto. Botucatu, 2010

\begin{tabular}{llll}
\hline & $\begin{array}{l}\text { ITU por 1000 } \\
\text { SVD-dia }\end{array}$ & $\begin{array}{l}\text { ICS por } 1000 \\
\text { CVC-dia }\end{array}$ & $\begin{array}{l}\text { Pneu VM por 1000 } \\
\text { VM-dia }\end{array}$ \\
\hline $\begin{array}{l}\text { Nov/2008- } \\
\text { Abr/2009 }\end{array}$ & 13,0 & 13,8 & 35,7 \\
Jun-Nov/2009 & 17 & 16,7 & 27,7 \\
$\begin{array}{l}\text { Legenda: ITU = Infecção do trato urinário, ICS = Infecção da corrente sanguínea; Pneu VM= Pneumonia associada à ventilação } \\
\text { mecânica }\end{array}$
\end{tabular}


Já na Tabela 5 evidencia-se que, apesar da variação da incidência global de Infecção Relacionada à Assistência à Saúde (IRAS), sendo a maior no mês de setembro e a menor no mês de outubro, o NAS médio permaneceu constante nos quatro meses analisados.

Tabela 5. Distribuição da incidência da taxa de ocupação, IRAS e NAS médio da UTI Adulto nos meses de julho a outubro de 2010. Botucatu, 2010

\begin{tabular}{lcccc}
\hline & JULH0 & AGOST0 & SETEMBRO & OUTUBRO \\
\hline Taxa de ocupação (\%) & 96,3 & 89,3 & 91,8 & 86 \\
Incidência global de IRAS(por 1.000 pacientes-dia) & 46,9 & 41 & 31,5 & 22,5 \\
NAS médio & 71,5 & 70,7 & 71,9 & 73,2 \\
\hline
\end{tabular}

\section{DISCUSSÃO}

A predominância de especialidades cirúrgicas nesse estudo difere-se de um trabalho realizado no hospital geral da rede privada, o qual apresentou a maioria de internações por especialidades clínicas. 0 sexo masculino, com o maior percentual, também foi encontrado como resultado em outros trabalhos ${ }^{1,9,10}$.

Como possibilidade de justificativa para esse fato, seria que a amostra deste estudo foi constituída predominantemente por pacientes neurocirúrgicos, incluindo-se nesse grupo as vítimas de trauma, com alta prevalência para jovens do sexo masculino.

Quanto a média de idade de 57 anos, outros trabalhos brasileiros apresentaram a média aproximada de idade de 53 anos $^{9}, 60$ anos $^{8}$ e 70 anos ${ }^{1,11}$.

Em trabalhos realizados em UTIs do município de São Paulo, a taxa de óbito foi de $25 \%{ }^{9}$ e de $38 \%{ }^{11}$. A taxa de $27 \%$ de óbito, evidenciada nesse trabalho, mesmo que entre a média dessas UTIs brasileiras, relaciona-se pela associação de diferentes fatores, como a presença de doenças crônicas preexistentes dos pacientes internados nessa UTI e a Infecção Hospitalar. 0 fato também de se tratar de um hospital-escola terciário que é referência para a região pode levar a concentração de casos mais graves e a admissões na UTI de pacientes fora de possibilidades terapêuticas.

Segundo levantamento da Associação de Medicina Intensiva Brasileira $(A M I B)^{12}$, a média de permanência de $69 \%$ dos pacientes nas UTIs brasileiras é de um a seis dias. A UTI do estudo, com o tempo médio de internação de nove dias, portanto, não se assemelha à realidade nacional. Infere-se por ser um hospital-escola referência e por contar, novamente, com a exacerbação da doença crônica preexistente e a Infecção Hospitalar.

Quanto a avaliação da carga de trabalho de enfermagem, nessa mesma UTI, no ano de 2009, o NAS médio foi menor, $60 \%$, variando de $28 \%$ a $123 \% 7$.

Também um trabalho que analisou a carga de trabalho de enfermagem em uma UTI geral de um hospital universitário de médio porte apresentou média do NAS de $66 \%$, mínima de $48 \%$ e máxima de $82 \% 6$. Em uma pesquisa realizada na UTI geral de um hospital público universitário, associando estilos de liderança e carga de trabalho de enfermagem com o NAS, mostrou que a média do escore foi de $80 \%$, com variação entre $62 \%$ e $102 \%$. Portanto, há índices elevados quando se consideram as necessidades de cuidados e, consequentemente, a carga de trabalho de enfermagem na UTI. Na literatura internacional, apenas dois estudos foram encontrados com base no NAS e seus escores médios foram menores, cerca de $41 \%^{13,14 .}$

As características dos pacientes, bem como a gravidade da doença, poderiam explicar a diferença de média NAS entre UTIs de diferentes países. Além disso, as dificuldades relacionadas para uma explicação clara sobre alguns itens operacionais do NAS poderiam contribuir para essas diferenças.

A real necessidade de recursos humanos para o serviço de enfermagem por meio do NAS fica demonstrada e pode mudar as práticas tradicionais de gerenciamento ainda adotadas na maioria dos hospitais, onde todos os pacientes são assistidos como se demandassem indistintamente a mesma quantidade de cuidados. Com isso, não se leva em conta que cada paciente se comporta subjetivamente de maneira diferente, exigindo da enfermagem uma assistência diferenciada em termos qualitativos e quantitativos.

No estudo realizado em um hospital universitário no município de São Paulo ${ }^{11}$, os pacientes que permaneceram internados na UTI entre 6 e 10 dias e mais do que 10 dias apresentaram maiores escores do NAS, respectivamente $73 \%$ e $72 \%$, quando comparados com permanência menor do que cinco dias, com score de $63 \%$, diferente do encontrado nesse estudo, no qual a correlação negativa demonstrou que os pacientes que apresentavam menor tempo de internação tinham maior carga de trabalho. Outro estudo recente realizado na Grécia ${ }^{15}$ mostrou que os pacientes que obtinham o primeiro score NAS acima de $61,6 \%$ apresentavam um risco 5,2 vezes maior de permanência em uma UTI de cirurgia cardíaca por mais de dois dias.

Quanto à idade dos paciente, foi visto que essa variável não tem influência na carga de trabalho de enfermagem na UTI, o mesmo encontrado em outros estudos brasileiros realizados em hospitais universitários ${ }^{10,11}$. Pelos resultados pode-se inferir que, independente da idade, investimentos 
terapêuticos são aplicados para promover a melhora clínica dos pacientes.

Os pacientes com o desfecho óbito foram os que apresentaram um maior NAS, o mesmo também evidenciado em outros estudos ${ }^{10,11}$. A alta mortalidade observada e a permanência prolongada dos pacientes confirmam a elevada necessidade de cuidados requeridos e a alta carga de trabalho de enfermagem nesta UTI.

Com relação as Infecções Relacionadas à Assistência à Saúde, principalmente as adquiridas no ambiente hospitalar, estão entre as principais causas de morbidade e de mortalidade, e, por consequência, há aumento do tempo de internação e de custo por paciente. Elas podem ser amenizadas e até mesmo evitadas com a aplicação de medidas de prevenção, adotadas antes mesmo da internação do paciente e que estejam baseadas em conhecimento técnico adequado e apoio administrativo ${ }^{16,17}$.

E nas UTIs dos hospitais-escola, as IH encontram-se ainda mais elevadas devido à pluralidade de doentes com diferentes diagnósticos, associados aos mais diversos níveis de gravidade e/ou complicações. Somam-se, ainda, equipamentos sofisticados, de alta tecnologia, teoricamente, maiores recursos terapêuticos, como biopsias, cateterizações, aspirações de fluidos, sem contar com o número de fômites, alunos e profissionais circulantes dentro das unidades de internaçãa ${ }^{17}$.

Trata-se de um desafio na busca pela manutenção da vida na qual a maioria absoluta dos pacientes é submetida a procedimentos invasivos que prejudicam os seus mecanismos de defesa; citam-se aqui o tubo orotraqueal, traqueostomia e ventilação mecânica, tendo como consequência a Pneumonia Associada à Ventilação Mecânica (PAVM), a sonda vesical de demora como causa para a infecção do trato urinário (ITU) e o cateter venoso central, principal responsável pela infecção de corrente sanguínea $(\mathrm{ICS})^{16}$.

Um estudo europeu foi desenvolvido para avaliar a influência do dimensionamento nas taxas de Infecções Hospitalares. A pesquisa constatou aumento de $50 \%$ de risco de infecção para pacientes atendidos por número de pessoal de enfermagem subestimado ${ }^{18}$.

A principal forma de reduzir a Infecção Hospitalar é por meio da realização de protocolos em conjunto com a Comissão de Controle de Infecções Relacionadas à Assistência à Saúde da instituição e com a educação permanente com a equipe; porém, ressalta-se que para esta equipe trabalhar com qualidade e segurança deve estar devidamente dimensionada para atender toda a demanda com o mínimo de risco ao paciente.

Diante desses resultados, vê-se que o compromisso das lideranças de enfermagem com a adequação do quadro de pessoal e seu impacto na organização do trabalho em saúde é crucial para o alcance de um cuidado individualizado, integral e seguro, e que não se pode adotar postura omissa diante dos riscos a que estão submetidos os pacientes diante de um quadro de pessoal inadequado.

\section{CONCLUSÃO}

A partir do exposto acima, pode-se concluir que a amostra constou principalmente de pacientes do sexo masculino e com idade média de 57 anos. As principais especialidades foram as cirúrgicas, principalmente neurocirúrgicas. 0 tempo de internação médio desses pacientes foi de 9 dias, e o principal desfecho foi a alta.

Quando comparados os índices de Infecção Hospitalar antes e após a implantação dos Sítios Assistenciais, viu-se que houve uma redução nas taxas de pneumonia associada à ventilação mecânica e um aumento nos índices de infecção urinária associado a sonda vesical de demora e infecção de corrente sanguínea associada ao cateter venoso central. Necessita-se de estudos mais específicos para concluir que a redução dos índices de PAVM ocorreu somente devido a nova organização da UTI em Sítios Assistenciais.

Em relação ao NAS, viu-se que o NAS médio da amostra foi de $72 \%$ variando de $48 \%$ a $109 \%$. Concluiu-se que, apesar da variação da incidência global de IRAS, sendo a maior no mês de setembro e a menor no mês de outubro, o NAS médio permaneceu constante nos quatro meses avaliados.

Destaca-se a importância em implantar novas formas de classificação de pacientes que atendam às necessidades conforme as realidades dos serviços em UTI, assim como foi proposto nesta pesquisa.

\section{REFERÊNCIAS}

1.Conish RMY, Gaidzinski RR. Nursing Activities Score-NAS como instrumento para medir carga de trabalho de enfermagem em UTI adulto. Rev Esc Enferm USP. 2007; 41(3): 346-54.

2.Vigna CP, Perroca MG. Utilização de sistema de classificação de pacientes e métodos de dimensionamento de pessoal de enfermagem. Arq Cienc Saude. 2007; 14(1): 8-12.

3.De Groot Há. Patient classification system evaluation. Part 2: System selection implementation. J Nurs Adm. 1989; 19(7): 24-30.

4. Tranquitelli AM, Padilha KG. Sistemas de classificação de pacientes como instrumentos de gestão em Unidades de Terapia Intensiva. Rev Esc Enferm USP. 2007; 41(1): 141-46.

5.Miranda DR, Raoul N, Rijik A, Schaufeli W, lapichino G. Nursing activities score. Crit Care Med. 2003; 31(2): 374-82.

6.Queijo AF, Padilha KG. Nursing Activities Score (NAS): adaptação transcultural e validação para a língua portuguesa. Rev Esc Enferm USP. 2009; 43(n esp): 1018-25. 
7.Castro MCN, Dell'Acqua MCQ, Corrente JE, Zornoff DCM, Arantes LF. Aplicativo informatizado com o Nursing Activities Score: instrumento para gerenciamento da assistência em unidade de terapia intensiva. Texto \& Contexto Enferm. 2009; 18(3): 577-85.

8.Dell'Acqua MCQ, Novelli e Castro MC, Arantes LF, Zornoff DCM, inventores. Score eletrônico de atividades de enfermagem em UTI. BR n.RS 11724-4. Botucatu: UNESP - depositante 1 set. 2007. [ citado 2011 set 13]

9.Balsanelli AP, Zanei SSV, Whitaker IY. Carga de trabalho de enfermagem e sua relação com a gravidade dos pacientes cirúrgicos em UTI. Acta Paul Enferm. 2006; 19(1): 16-20.

10.Gonçalves LA, Padilha KG. Fatores associados à carga de trabalho de enfermagem em Unidade de Terapia Intensiva. Rev Esc Enferm USP. 2007; 41(4): 645-52.

11.Gonçalves LA, Garcia PC, Toffoleto MC, Telles SCR, Padilha KG. Necessidades de cuidados de enfermagem em terapia intensiva: evolução diária dos pacientes segundo o NAS. Rev Bras Enferm. 2006; 59(1): 5660.

12.Associação de Medicina Intensiva Brasileira- AMIB. Anuário Brasileiro de Unidades de Terapia Intensiva. v.1.São Paulo; 1999/2000.

13.Adell AB, Campos RA, Cubedo RM, Quintana BJ, Sanahuja RE, Sanchís MJ, et al. Nursing Activity Score (NAS). Our experience with a nursing load calculation system based on times. Enferm Intens. 2005; 16(4): 164-73.

14. Adell AB, Campos RA, Bou MY, Bellmunt JQ, Garcia CG, Canuto MS, et al. Care workload in critical patients: comparative study NEMS versus NAS. Enferm Intens. 2006; 17(2): 67-77.

15.Giakoumidakis K, Baltopoulos Gl, Charitos C, Patelarou E, Galanis P, Brokalaki H. Risk factors for prolonged stay in cardiac surgery intensive care units. Nurs Crit Care. 2011; 16(5): 243-51.

16.Pombo CMN, Almeida PC, Rodrigues JLN. Conhecimento dos profissionais de saúde na Unidade de Terapia Intensiva sobre prevenção de pneumonia associada à ventilação mecânica. Cienc Saude Colet. 2010; 15(supl 1): 1061-72.

17.Aguiar DF, Lima ABG, Santos RB. Uso das precauções padrão na assistência de enfermagem: um estudo retrospectivo. Esc Anna Nery. 2008; 12(3): 571-75.

18. Hugonnet S, Chevrolet JC, Pittet D. The effect of workload on infection risk in critically ill patients. Crit Care Med. 2007; 35(1): 76-81. 